\title{
Intravascular Ultrasound (IVUS)
}

S MOKADDAS HOSSAIN (SADI)

Labaid Cardiac Hospital, Dhaka, Bangladesh

Address of Correspondence: Dr. S Mokaddas Hossain (Sadi), Associate Professor (Cardiology), Email:s_mokaddas_hossain@yahoo.com

\section{Introduction:}

Intravascular Ultrasound (IVUS) imaging is a clinical method to directly visualize atherosclerosis and other pathologic conditions within the walls of blood vessel. This system uses higher frequencies than noninvasive echocardiography achieving greater radial resolution. Current IVUS catheter uses frequencies from 20 to $30 \mathrm{MHZ}$. Although coronary angiography is considered standard for coronary artery imaging, it has several inherent limitation. Angiography detects luminography, it reveals little else about atherosclerotic plaque. When normal proximal reference segment does not exists,as in diffuse atherosclerosis, Left main aorto ostio proximal disease, it is difficult to detect and quantitate atherosclerosis with angiography. Angiography also underestimate atherosclerosis in case of remodeling artery. Due to improve quality of stent, routine use of IVUS guided stent deployment is not superior to angiography. However IVUS guided approach may reduce target vessel revascularization and can detect stent abnormality (expansion, apposition, edge dissection, instent restenosis etc) that are not detected well by angiography.

The first true IVUS system was designed by bom and his associates in Rotterdam in 1971. ${ }^{1.2}$ an improved technique for visualizing cardiac chambers and valves. The first transluminal images of human arteries were recorded by yock and his associates in $1988 .^{3}$

\section{Basic of IVUS}

Ultrasound is just a high frequency form of sound. Audible Sound $20-20,000 \mathrm{~Hz}$. Ultrasound $>20,000 \mathrm{HZ}$ is high frequency sound waves. Medical Ultrasound is typically 1-50 MHZ. 1 MHZ=1,000,000 cycles/second. Higher frequency gives more resolution. Resolution occur in axial and lateral direction.

Currently there are two basic technical approaches to catheter based ultrasound Imaging. Mechanical (rotating) solid state, synthetic phase electronic array. ${ }^{4}$ Mechanical and synthetic phased array transducer are used. Monorail (Rapid Exchange) and OTW catheter are used. Compatible guide wires are $0.018 " / 0.014 "$. Two types Machine are common use, Galaxy (Boston Scientific) and Volcano. (Fig: 1) For Galaxy, Ultracross Atlantis Guide Catheter and for volcano eagle eye guide are usually used. By using both system the Ultrasound beam is rotated at approximately 1800 rpm.

Functionally non - LMCA stenosis of a major epicardial artery MLA $<4$ sq mm (MLA 2.4 sq mm, corresponding FFR $<0.80$ ) is significant. ${ }^{5}$ (Fig: 2a)

A left main cross sectional area MLA $<6$ sq mm (MLA 4.8 sq mm, corresponding FFR $<0.80$ ) new IVUS criteria for functionally significant LMCA stenosis. ${ }^{6}$ (Fig:2b)

The recognition of imaging artifacts is critical in order to avoid image misinterpretation. Ring down shadow, NURD (Non-Uniform Rotational Distortion Fig: 9), side lobes vision, Reverberations, ghosts shadow etc may create troubleshooting in IVUS image interpretation. ${ }^{7}$

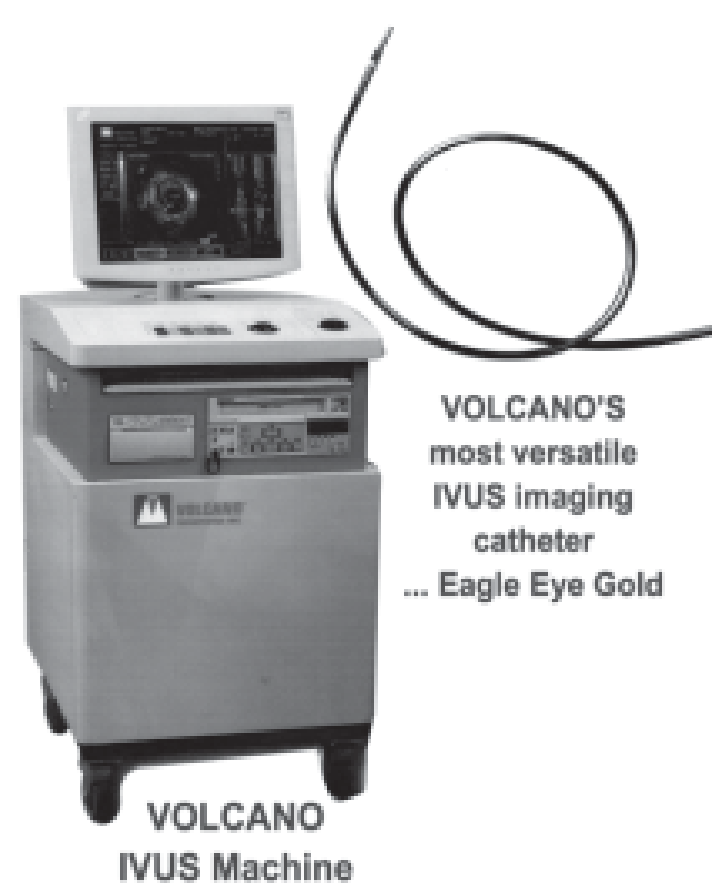

Fig.-1: Volcano IVUS Machine \& Eagle Eye Catheter 


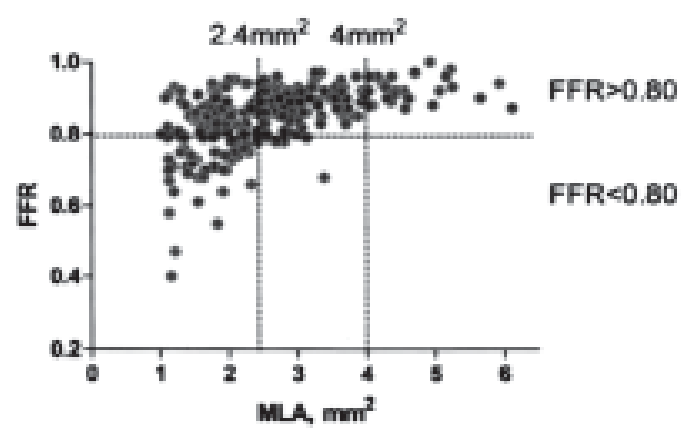

Fig.-2a. New IVUS Criteria for Functionally significant Non-LMCA Stenosis

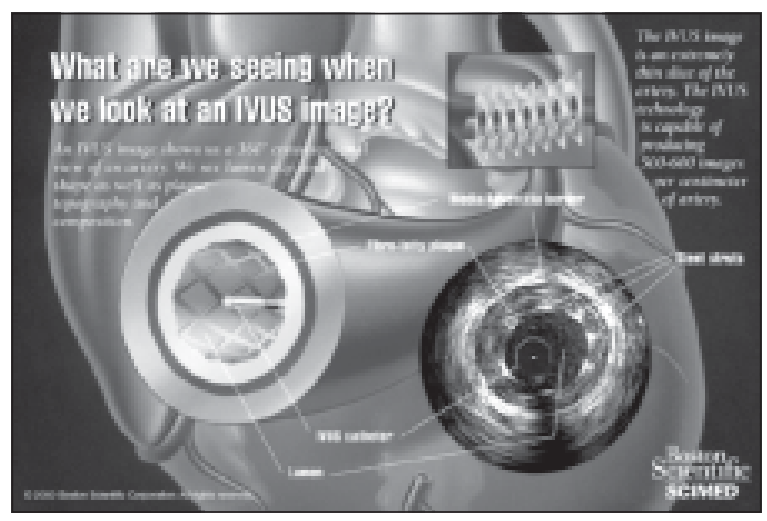

Fig.-3: Inside Information of Coronary Artery by IVUS

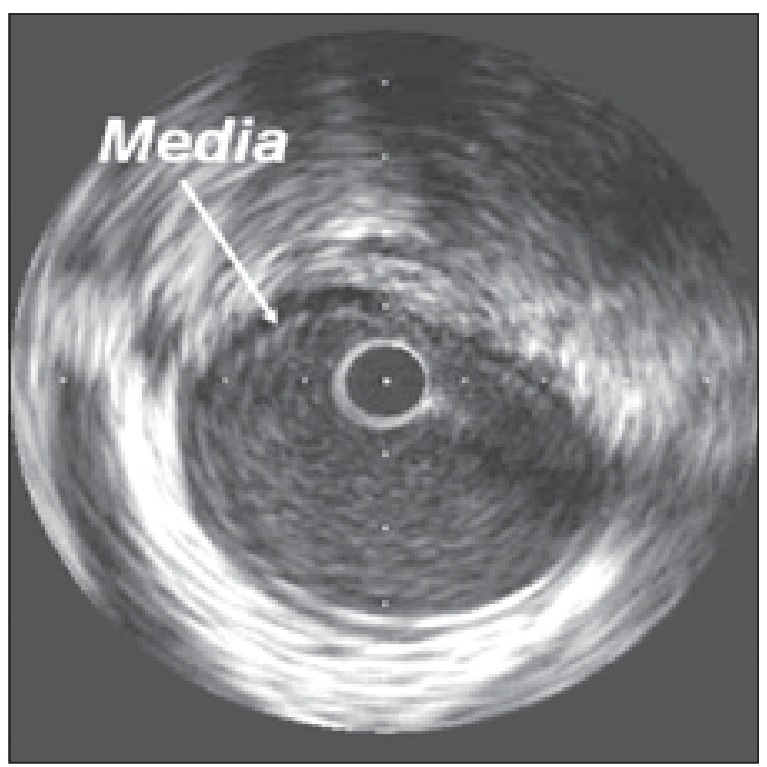

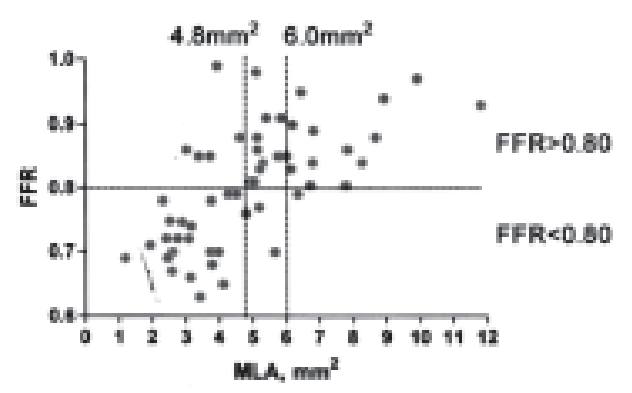

Fig.-2b. New IVUS Criteria for Functionally significant LMCA Stenosis

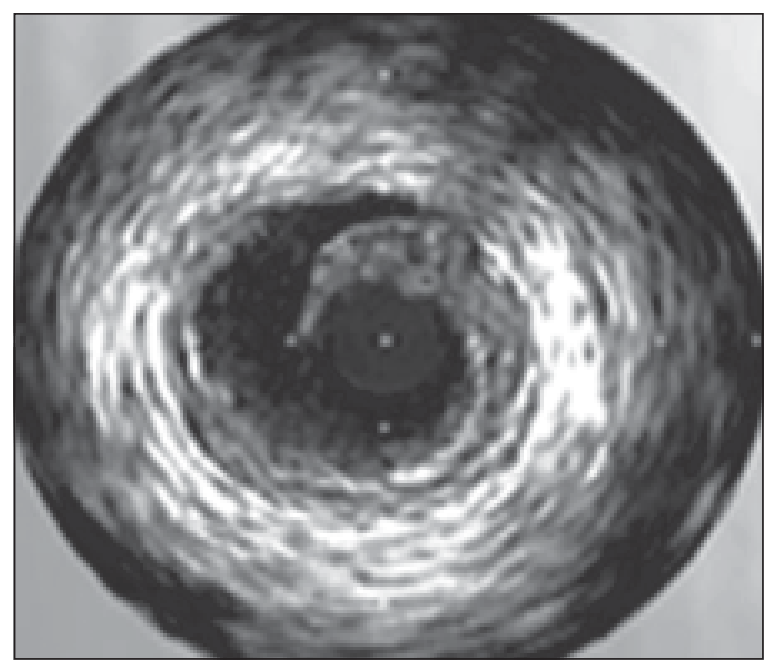

Fig.-4: Deep fibro-fatty plaque dissection extending into the media

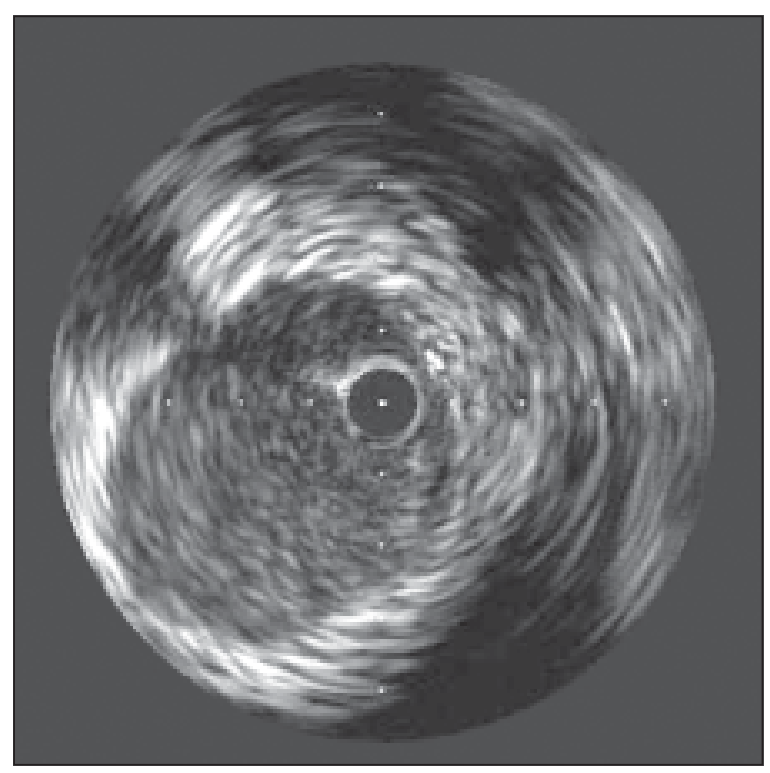

IVUS Picture of

Fig.-5a: Left Main Coronary Artery

Fig.-5b: Vein 


\section{QuantitativeAnalysis}

During clinical IVUS imaging of a non stented lesions, two distinct boundaries that have consistent histologic correlation: the lumen intima (or lumen-plaque) Interface and media adventitia Interface. Thus in a non-stented vesel two cross sectional area, EEM CSA and Lumen CSA. In slented vessels three boundaries: Lumen CSA, stent CSA and EEM CSA. ${ }^{8}$ (Fig: 3 )

The first "structure" that the IVUS beam sees is the blood filled lumen. Blood has a speckled and continuously changing pattern that is distinct from tissue. Lumen measurements are performed at the interface between the lumen and the leading edge of the intima relative to the center of mass of the lumen.

\section{Intracoronary Stents}

Endovascular stents are intensely echo reflective. (Fig: 6) The metallic prosthesis creates a third IVUS boundary between the lumen and EEM. Stent CSA and intimal hyperplasia can be measured. Intimal hyperplasia can be calculated as stent CSA minus lumen CSA. Stent apposition refers to the proximity of struts to the arterial wall and preclude blood flow between strut and under lying wall. Apposition is different from expansion and the two terms should not be used interchangeably. Malaposition (or Incomplete apposition) is defined as separation of $\geq 1$ stent struts from the intima. (Fig: 7)

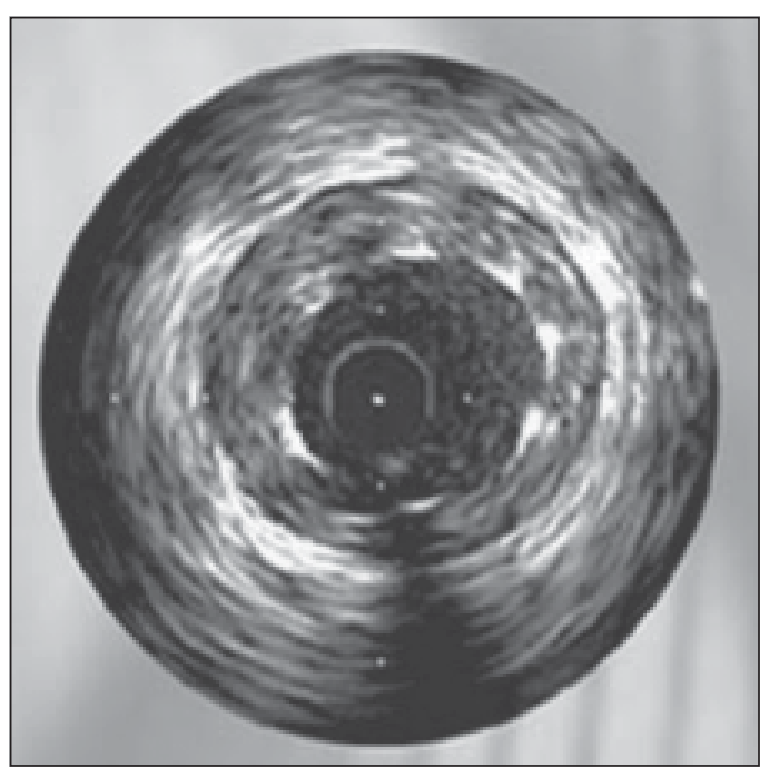

Fig.-6: Fully apposed palmaz-schatz stent

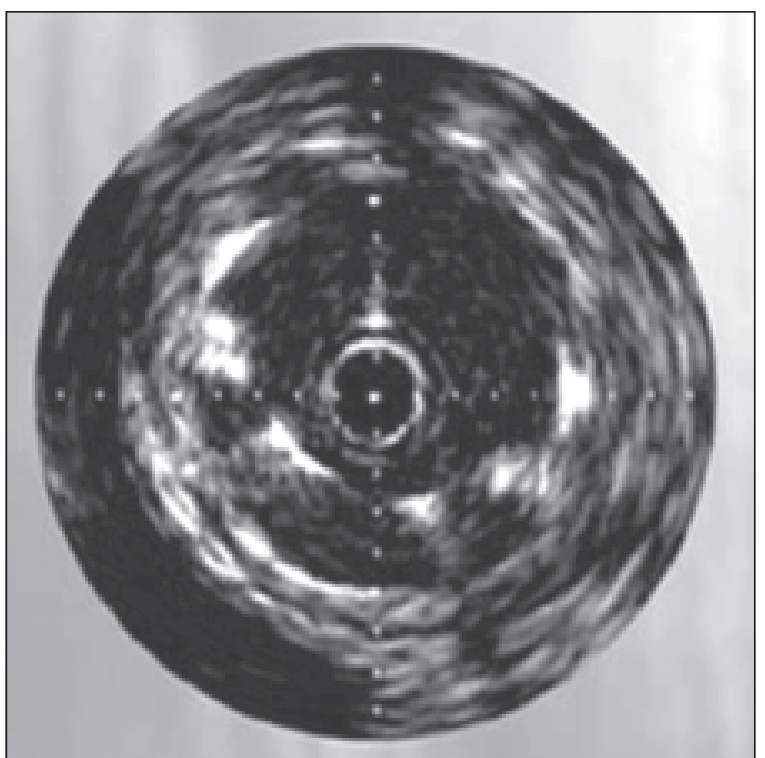

Fig.-7: Incompletely apposed palmaz-schatz stent

Post-intervention-in the absence of tissue prolapse or stent malapposition the stent CSA will be equivalent to the lumen CSA. Measurements include stent CSA, minimum stent diameter, Maximum stent diameter, stent symmetry (ratio of minimum to maximum stent diameter), and stent expansion (the minimum stent CSA compared with a predefined reference, which can be the proximal, distal, largest or average reference lumen CSA, or even the lesion or reference EEM CSA).

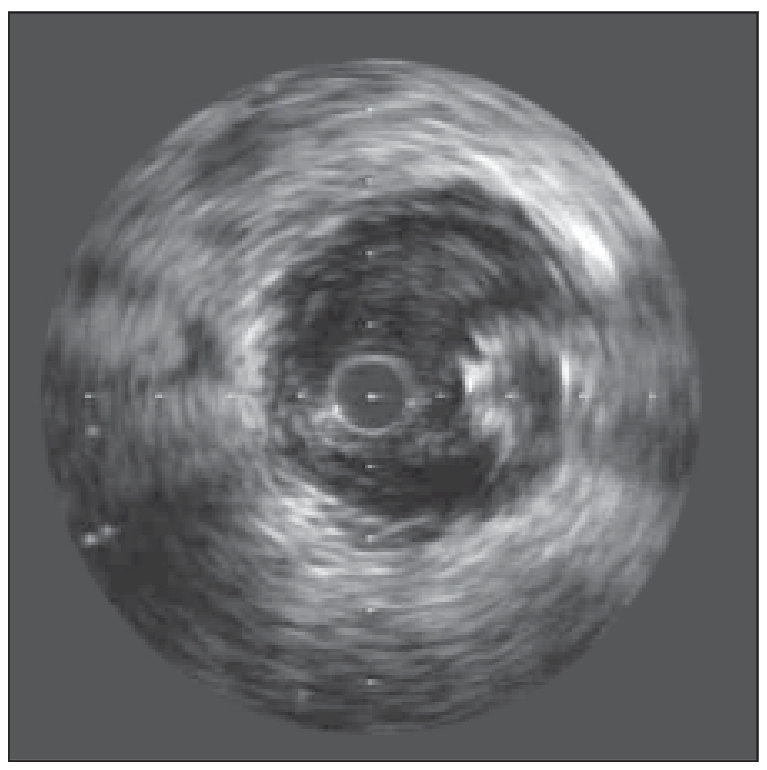

Fig.-8: Crushed stent 


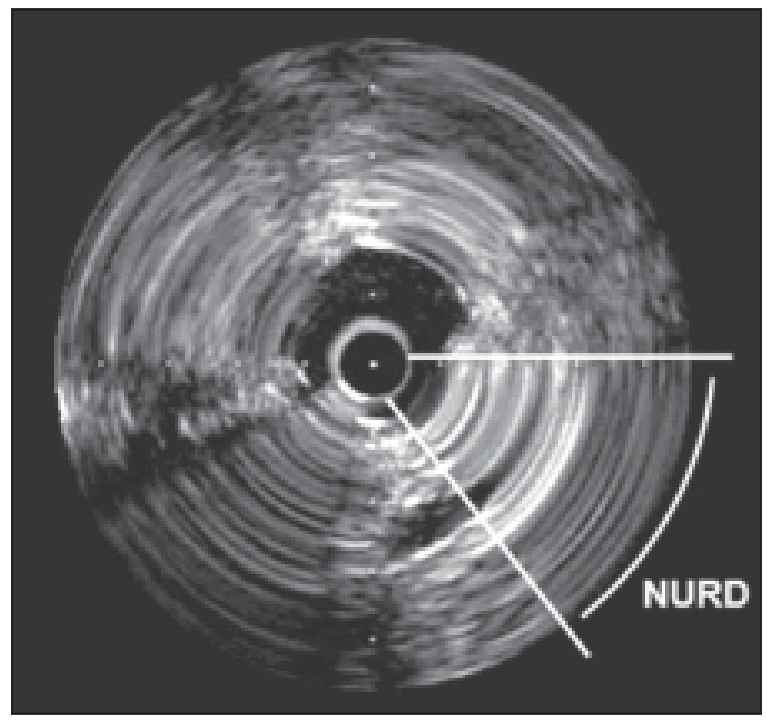

Fig.-9: Catheter Bending

\section{Saphenous Vein Grafts}

In situ veins do not have an EEM. (Fig:5b) However, saphenons vein grafts typically undergo arterialization with morphologic changes that include intimal fibrous thickening, medial hypertrophy and lipid deposition to create and echolucent zone. ${ }^{7}$ The EEM CSA which is actually the vein graft CSA is measured by tracing the outerborder of this echolucent zone. All other measurement including "P \& M" CSA and Plaque burder, are calculated in a similar manner to native coronary disease. ${ }^{10,11}$

\section{Plaque Composition}

The threshold between normal and abnormal is the subject of some debate but more than $0.3 \mathrm{~mm}$ of intimal thickening is probably abnormal and can be used to distinguish from atherosclerosis. Atherosclerotic lesion are heterogenous and include varying amounts of calcium, dense fibrous tissue, lipid, smooth muscle cells, thrombus etc. By IVUS imaging can grossly separate lesions into subtypes according to echodensity and presence or absence of shadowing and reverberations. ${ }^{12}$

\section{Virtual Histology}

True histological level plaque classification may be possible with IVUS tissue characterization that also employ radiofrequency domain information. ${ }^{13}$ Using the advanced radiofrequency analysis, VH-IVUS classifies plaque into four major components: fibrous (dark green), fibrofatty (yellow-green), necrotic core (red), and dense calcium (white) ${ }^{13,14,15}$ (Fig:10)

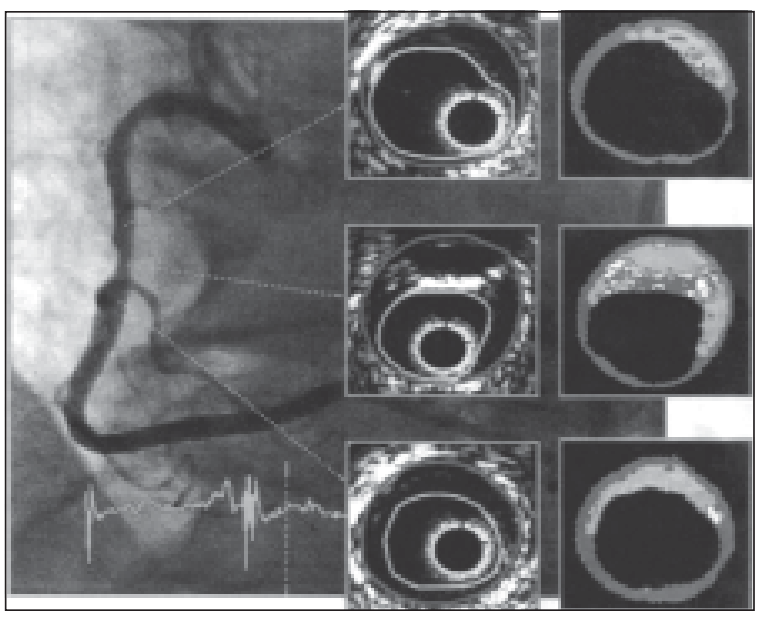

Fig.-10: VH-IVUS Imaging

\section{Calcium}

Calcium is a powerful reflector of ultrasound. In practice calcium is echo dense (hyperechoic) plaque (brighter than the reference adventitia) that shadows using IVUS calcium can be localized and characterized as superficial (closer to tissue-lumen interface) and deep (closer to the mediaadventitia junction) and quantified according to its arc and length. (Fig. 11,12,13)

\section{Fibrous Plaques}

Fibrous plaques represents the majority of atherosclerotic lesion. Very dense fibrous plaques may produce sufficient

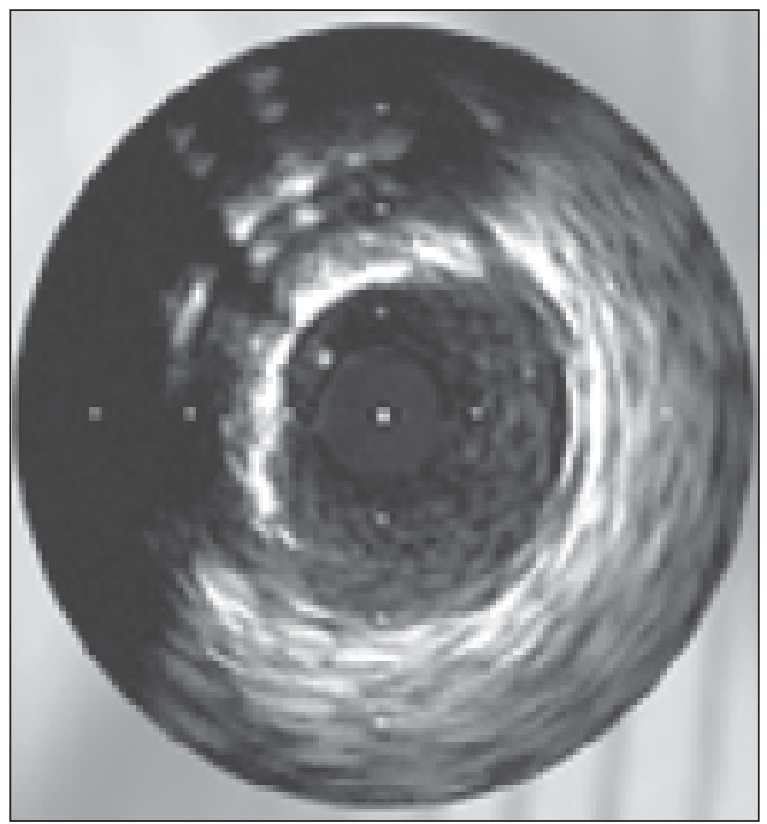

Fig.-11: $180^{\circ}$ arc of eccentric superficial calcified plaque 


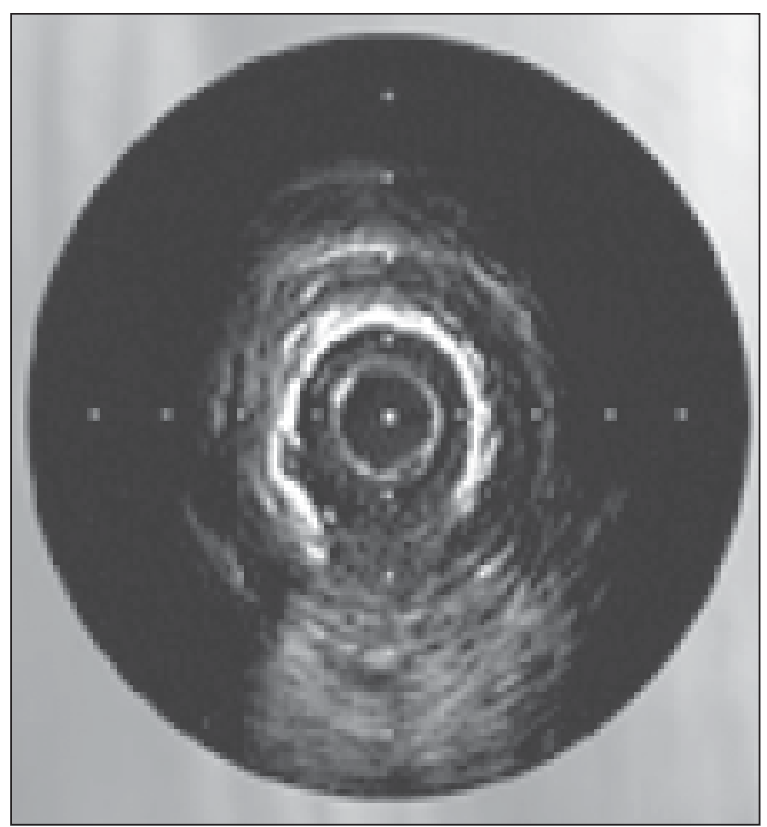

Fig.-12: $270^{0}$ arc of eccentric superficial calcified plaque

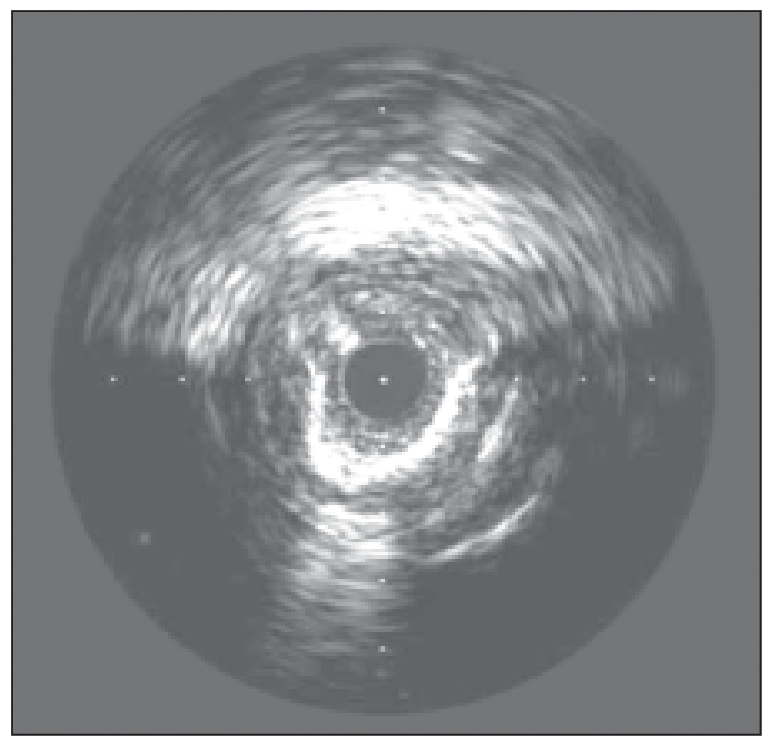

Fig.-13: Superficial Calcium needs Rotational Atherectomy for treatment

attenuation to be misclassified as calcification with acoustic shadowing. Both calcified and fibrotic plaque are hyperechoic. (Fig. 4,14)

\section{Soft Plaques}

Echo lucent Plaque (hypoechoic) are less bright compared with reference adventitia. Echolucent (soft) plaque contain varying amounts of fibrous and fatty tissue. Reduced

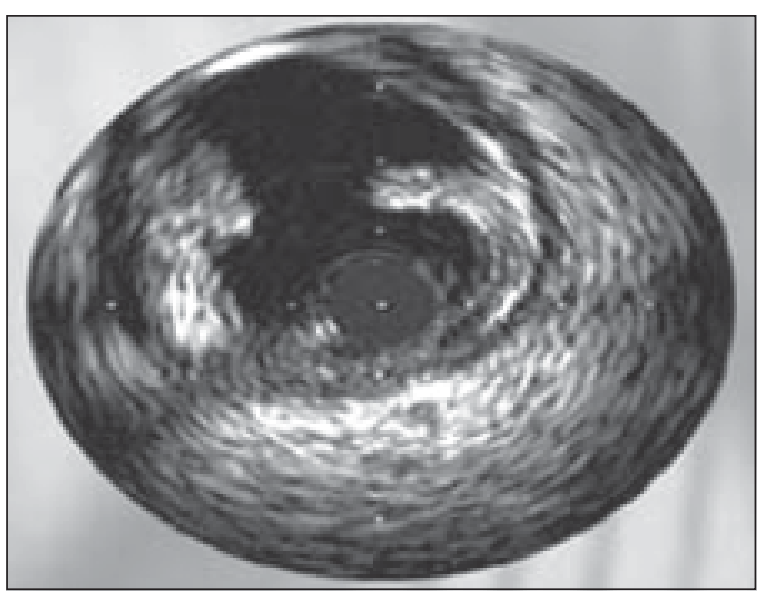

Fig.-14: Fibrous Plaque dissection extending into the intima

echogenicity may also result from a necrotic zone within the plaque, an intramural hemorrhage, or a thrombus (Fig: 17).

Mixed plaque also called “fibrocalcific”, "fibrofatty” plaque.

\section{Intimal Hyperplasia}

The neointimal hyperplasia of early in-stent restenosis often appears to have low echogenecity. The intimal hyperplasia of late in-stent restenosis may appear more echogenic. (Fig: 15)

\section{Thrombus}

The identification of thrombus is one of the most difficult aspects of IVUS imaging. ${ }^{16,17}$ clues to the presence of thrombus include the following

1) Sparkling "Scintillating” appearance

2) Lobulated mass projecting into the lumen

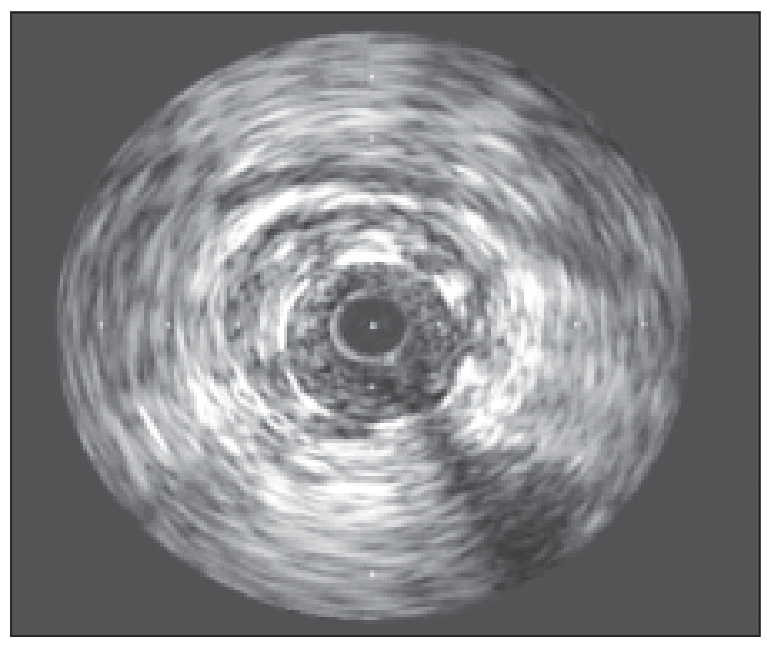

Fig.-15: Inadequate stent deployment \& Neointimal hyperplasia 
3) A distinct interface between the suspected thrombus and under lying plaque.

4) Identification of blood speckle within the thrombus indicating microchannels through the thrombus.

5) Mobility

Routine IVUS diagnosis of thrombus in native coronary arteries is difficult, (Fig: 16, 17) the diagnosis of thrombus in saphenous vein graft is frankly totally unreliable

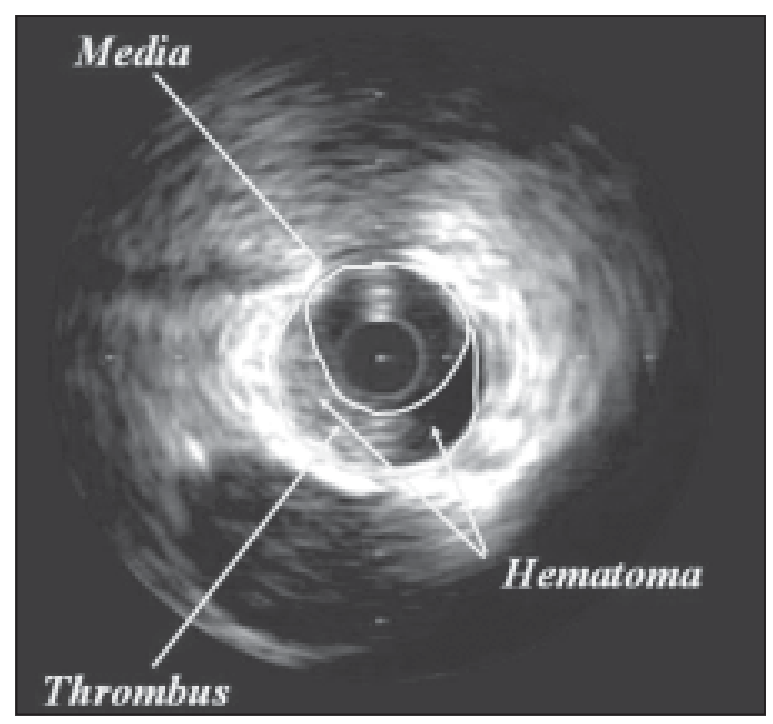

Fig.-16: Hematoma \& Thrombus

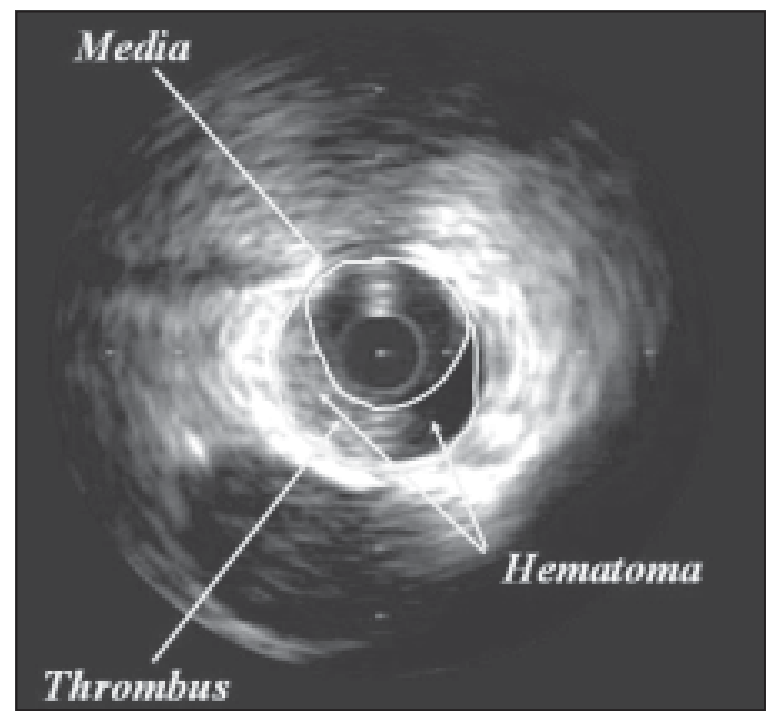

Fig.-17: Ruptured plaque presenting with acute coronary sundrome

\section{Conclusion:}

In the stent era, IVUS qualitative morphology assessment is less important than quantitative measurements for proper stent sizing (diameter and length). The IVUS minimum lumen area correlated strongly with Doppler flow wire coronary flow reserve, pressure- wire fractional flow reserve and exercise thallium. A minimum lumen CSA of less than $4 \mathrm{~mm}^{2}$ correlate with ischemia and indicate worse patient outcome. However this cutoff applies only to major epicardial vessels, excluding left main coronary artery, saphenous vein grafts and small arteries (branches and distal vessel)

In general there are two criteria that have been used to perform revascularization for left main disease: either a minimum lumen CSA below $5.5-6 \mathrm{~mm}^{2}$ or an IVUS diameter stenosis greater than $50 \%$ compared with the reference lumen dimension. In general, when a parent artery bifurcate into two daughter arteries, the sum of the two daughter arteries approximately 1.5 times the size of the parent. The left main disease is frequently diffuse with no true reference segment (producing the so called "small left man"), measurement of absolute left main lumen dimension is preferable.

\section{References:}

1. Bom N, lencee CT, Van Egmond FC, An Ultrosonic intracardiac scanner, Ultrasonics 1972;10:71-6

2. Bom N, Ten Hoff H, Lancee CT, et al. Early and recent intraluminal ultrasound devices. Int J Card imaging 1989; 4: 79-88.

3. Yock PG, Johnson EL, Linker DT. Intravascular ultrasound: development and clinical potential. AMJ Card image 1988;2: 185-93

4. Forst S, Freeman NA, Johnston P, et al. In vitro and In vivo comparison of three different intravascular ultrasound catheter designs. Catheter cardiovasc interv 2001; 52:382-92

5. Kang Sj, LeeJY AhnJM, MINTZ GS, Kim WJ, Park DW, PARK SJ et al. Validation of Intravascular Ultrasound-derived parameter with fractional flow reserve for assessment of coronary stenosis severity. Circ Cardiovasc Interv. 2011;4:6571.

6. Kang Sj, LeeJY AhnJM, MINTZ GS, Kim WJ, Park DW, PARK SJ et al. Intravascular Ultrasound-derived predictor for fractional flow reserve in intermediate left main disease. JACC cardiovasc interven. 2011;4:1168-1174.

7. Mintz Gs. Basics. Mintz S Gary. Editor Intracoronary Ultrosound. First Edition 2005: 1-18

8. Mint Z Gs. Quantitative and qualitative analyses. Mintz S Gary. Editor Intracoronary Ultrasound. First Edition 2005:1943

9. Spray TL, Roberts WC. change in saphenous veins used as aorto coronary by pass grafts. Am Heart J 1977; 500-16

10. Jain SP, Roubin Gs, Nanda Nc, et al. Intravascular ultrasound imaging of saphenous vein graft stenosis. Am J cardiol 1992; 69:133-6 
11. Hong M-K, Mintz Gs, Hong MK, et al. Intravascular ultrasound assessment of the presence of vascular remodelling in diseased human saphenous vein by pass grafts. Am J Cardiol 1999 ; 84:992-8

12. Tobis JM, Mallery J, Mahon D, et al. Intravascular ultrasound imaging of human coronary arteries in vivo. Analysis of tissue characterization s with comparison to in vitro histological specimems. Circulation 1991;83:913-26

13. Nair A. Kubon BD, Tuzcu EM, et al. Coronary plaque classification with intravascular ultrasound radiofrequency data analysis. Circulation 2002;2200-6

14. Scott DS, Arora UK, Farb A, et al. Pathologic Validation of a new method to quantify coronary calcific deposits in vivo using intravascular ultrasound. Am J cardiol 2000;85:37-40
15. Seung-Jung Park, Cheol Whan Lee, Young-Hak Kim, SeungWhan Lee, Soo-Jin Kang et al. New Plaque imaging: Virtual Histology-Intravascular Ultrasound and Optical Coherence Tomography. Editor Seung-Jung Park, Cheol Whan Lee, Young - Hak Kim, Seung- whan Lee. COMPLEX ANGIO PLASTY, Fifth Edition. 2012:47-55.

16. Chemarin- Alibelli MJ, Pieraggi MT, Elbar (Elbaz) M, et al. Identification of coronary thrombus after myocardial infarction by intracoronary ultrasound compared with histology of tissues sampled by atherectomy. AM J Cardiol 1996; 77:344-9

17. Frimerman A, Miller HI, Hallman M, et al. Intravascular ultrasound characterization of thrombi of different composition. Am J Cardiol 1994; 73:1053-7 